\title{
Exploiting the features of energy-dispersive synchrotron diffraction for advanced residual stress and texture analysis
}

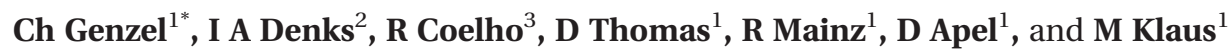 \\ ${ }^{1}$ Helmholtz-Zentrum Berlin für Materialien und Energie, Berlin, Germany \\ ${ }^{2}$ Salzgitter Mannesmann Forschung GmbH, Germany \\ ${ }^{3}$ Technische Universität Berlin, Berlin, Germany
}

The manuscript was received on 29 October 2010 and was accepted after revision for publication on 23 February 2011.

DOI: $10.1177 / 0309324711403824$

\begin{abstract}
Responding to a growing interest from the materials science community for residual stress, texture, and microstructure analysis, strong efforts are made to enhance existing and develop novel methods that allow for fast in-situ studies at elevated temperature, measurements under external load, or residual strain, and stress scanning with high spatial resolution. In the paper, energy-dispersive diffraction using high-energy white synchrotron radiation is shown to provide some distinct advantages concerning residual stress and texture analysis, which mainly arise from the fact that the energy-dispersive diffraction mode allows for the measurement of complete diffraction patterns under fixed but arbitrary scattering angles, $2 \theta$. A new two-detector set-up for simultaneous in- and out-of-plane diffraction analysis, which has been put into operation recently at the energy-dispersive materials science beamline EDDI at BESSY II, is introduced by using the examples of real-space residual stress and texture depth profiling on mechanically treated polycrystalline materials as well as of the in-situ study of (residual) stress evolution in a thin film at elevated temperature. It will be demonstrated that the individual measuring problems require the application of different geometrical slit configurations to define the pathways of the diffracted beams.
\end{abstract}

Keywords: energy-dispersive diffraction, residual stress, texture, depth profiling, in-situ thinfilm characterization

\section{INTRODUCTION}

Although the first x-ray diffraction (XRD) experiment carried out by Laue, Friedrich,and Knipping in 1912 was a white beam - and therefore an energy selective - experiment, it was more than half a century before the method of energy-dispersive (ED) diffraction was introduced in 1968 independently by Giessen and Gordon [1] and Buras et al. [2]. The reason for this delay was due to the fact that $\mathrm{ED}$ diffraction requires appropriate solid state detector systems with efficient photon energy resolution, which were not available

*Corresponding author: Helmholtz-Zentrum Berlin für Materialien und Energie, Lise-Meitner-Campus, Hahn-MeitnerPlatz 1, Berlin 14109, Germany. email: genzel@helmholtzberlin.de in the early years of XRD. Whereas most of the progress made in the first decade after the introduction of the ED method was achieved by employing conventional $\mathrm{x}$-ray sources [3], the modern third-generation synchrotron radiation facilities available today provide much better possibilities for advanced white beam diffraction experiments.

Perhaps the most striking advantage of ED diffraction on polycrystalline materials compared with angle-dispersive (AD) diffraction is the fact that it allows the detection of complete diffraction patterns under fixed but arbitrary scattering directions, $2 \theta$. Therefore it is not surprising that the initial focus of ED diffraction experiments was on research fields in physics, chemistry, and materials sciences, which require a multitude of diffraction lines $E^{h k l}$ such as crystal structure determination [4], texture [5] and 
line profile analysis [6], or quantitative phase analysis [7]. Also experiments using complex sample environments like high-pressure cells, where the angular range for observing the diffracted beam is strongly limited by the apparatus used for sample processing, were reported in the early days of ED diffraction $[\mathbf{8}]$.

The first ED x-ray stress analysis (XSA) experiments were performed in 1977 [9]. Since that time numerous efforts have been made to develop and improve methods for ED residual stress analysis, and to profit from the special features provided by the ED mode of diffraction, see for example references $[\mathbf{1 0}]$ to $[\mathbf{1 3}]$. However, most XSA experiments are still performed in the $\mathrm{AD}$ diffraction mode with monochromatic radiation. This might be due to the fact that only very few (high-energy) white beam synchrotron beamlines exist, which are designed for the special demands of residual stress analysis. In this paper, results on residual stress and texture measurements carried out at the materials science beamline EDDI for energy-dispersive diffraction at BESSY II are presented. With a usable energy range between about $8 \mathrm{keV}$ and $120 \mathrm{keV}$ it is dedicated to the study of structure gradients in the outer regions of surface treated [14] and coated [15] polycrystalline materials, but also to texture depth profiling [16] and in-situ analysis of phase transformations $[\mathbf{1 7}]$ and micro-structural changes $[\mathbf{1 8}, \mathbf{1 9}]$ as well as to chemical reactions during thin-film processing [20].

This paper deals with lattice strain, texture, and residual stress analysis based on ED diffraction measurements in different directions with respect to the sample reference system. However, in contrast to conventional XSA experiments, the azimuth orientation and inclination of the diffraction vector was not varied by rotating and/or tilting the sample, respectively, but by a horizontal translation of the ED detector out of the vertical diffraction plane. The paper is organized as follows. After a brief introduction to ED x-ray stress analysis in section 2, the experimental set-up used for the investigations is outlined in section 3. Results of residual stress and texture analysis achieved by applying different configurations of the two-detector set-up are presented and discussed in section 4. Finally, some concluding remarks are given in section 5 .

\section{BASICS IN ENERGY DISPERSIVE X-RAY STRESS ANALYSIS}

In ED diffraction using a white x-ray beam with a continuous energy spectrum, the Bragg angle, $\theta$, and the diffraction angle, $2 \theta$, under which diffraction patterns are measured, can be chosen freely and remain fixed during the measurement. The relation between the lattice spacing $d^{h k l}$ and the corresponding reflection $E^{h k l}$ on the energy scale follows by combining the energy relation $E=h v=h \mathrm{c} / \lambda$ (where $h$ is Planck's constant, $c$ is velocity of light in a vacuum) and the Bragg equation. In $\boldsymbol{Q}$-space one obtains

$$
\left|\mathbf{Q}^{h k l}\right|=\frac{2 \pi}{d^{h k l}}=\frac{4 \pi}{h c} \sin \theta E^{h k l}=\text { const } \cdot E^{h k l}
$$

XSA investigations in the near-surface region of quasi-isotropic polycrystalline materials are based on the measurement of reflections $\mathrm{hkl}$ and the evaluation of lattice strains $\varepsilon_{\varphi \psi}^{h k l}=\left(d_{\varphi \psi}^{h k l}-d_{0}^{h k l}\right) / d_{0}^{h k l}$ (where $d_{0}^{h k l}$ is strain-free lattice spacing) for different azimuth and inclination angle sets $(\varphi, \psi)$ of the diffraction vector $\boldsymbol{g}^{h k l}$ with respect to the sample reference system $\boldsymbol{S}$ (Fig. 1).

Assuming a biaxial residual stress state of rotational symmetry (i.e. independent of $\varphi$ ), where $\sigma_{11}^{S}=\sigma_{22}^{S}=\sigma_{\|}^{S}$ and $\sigma_{i 3}^{S}=0(i=1,2,3)$ holds within the probed sample volume (the latter condition requires the absence of lateral gradients of the remaining stress components), the relation between the elastic lattice strain $\varepsilon_{\psi}^{h k l}=\left\{\varepsilon_{33}^{L}\right\}_{\psi}^{h k l}$ obtained for the diffracting crystallites and the average mechanical stress $\left\langle\sigma_{\|}^{S}\right\rangle$ of all crystallites in this volume fraction is given by

$$
\varepsilon_{\psi}^{h k l}=\left\{\varepsilon_{33}^{L}\right\}_{\psi}^{h k l}=\left(\frac{1}{2} S_{2}^{h k l} \sin ^{2} \psi+2 S_{1}^{h k l}\right)\left\langle\sigma_{\|}^{S}\right\rangle
$$

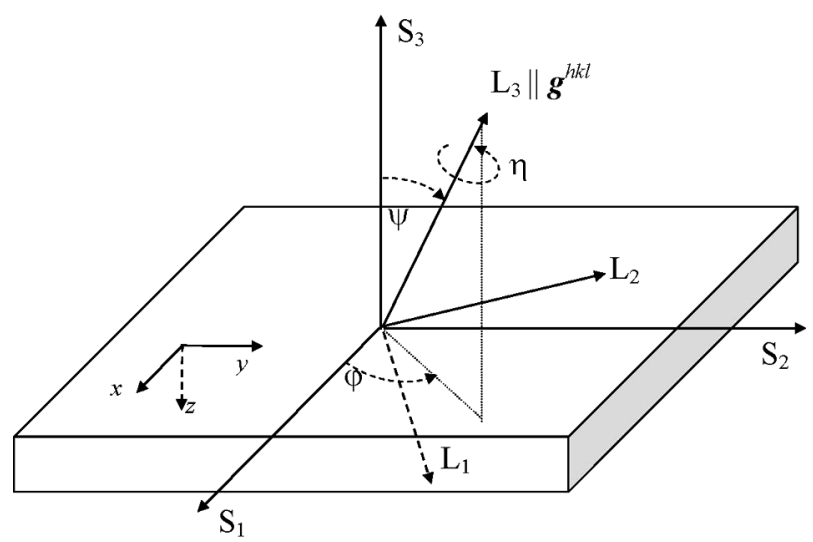

Fig. 1 Diffraction geometry in XSA. $\boldsymbol{S}$ and $\boldsymbol{L}$ denote the sample reference and the laboratory system, respectively. The positive $z$-axis of the sample system is directed towards the interior of the material. The angle set $(\varphi, \psi)$ defines the orientation of the diffraction vector $\boldsymbol{g}^{h k l}$ with respect to $S$, whereas $\eta$ describes the rotation of the sample around $\boldsymbol{g}^{h k l}$ 
where $S_{1}^{h k l}$ and $\frac{1}{2} S_{2}^{h k l}$ are the diffraction elastic constants (DEC) being valid for quasi-isotropic polycrystalline materials with random texture, which reflect the elastic anisotropy of the crystallites the material consists of. The superscripts $L$ and $S$ which indicate tensor components relating to the laboratory and the sample reference system, respectively, are omitted in the following. With equation (1) the lattice strain becomes a function of energy

$$
\varepsilon_{\psi}^{h k l}=\left\{\varepsilon_{33}^{L}\right\}_{\psi}^{h k l}=\frac{d_{\psi}^{h k l}}{d_{0}^{h k l}}-1=\frac{E_{0}^{h k l}}{E_{\psi}^{h k l}}-1
$$

with $E_{0}^{h k l}$ being the energy that corresponds to the strain-free lattice spacing $d_{0}^{h k l}$. If the lattice strains and stresses vary as functions of the depth, $z$ below the surface, equation (2) becomes

$$
\varepsilon_{\psi}^{h k l}(z)=\left(\frac{1}{2} S_{2}^{h k l} \sin ^{2} \psi+2 S_{1}^{h k l}\right) \sigma_{\|}(z)
$$

Since polycrystal diffraction methods always require a sufficiently large sampling volume defined by beam limiting slits or masks to assure satisfactory crystallite statistics, the real- or $z$-space profiles cannot be measured directly, but have to be evaluated from the experimentally obtained depth profiles by rather complex mathematical formalisms like inverse Laplace transform [21] and/or deconvolution procedures [22]. A general formulation for the information depth, to which the measured diffraction signal has to be assigned, is given by

$$
\langle z\rangle=\frac{\iiint_{\mathrm{GV}} z w(x, y, z, \theta, \psi, \eta) \mathrm{e}^{-\mu(E) k(\theta, \psi, \eta) z} \mathrm{~d} x \mathrm{~d} y \mathrm{~d} z}{\iiint_{V_{\mathrm{GV}}} w(x, y, z, \theta, \psi, \eta) \mathrm{e}^{-\mu(E) k(\theta, \psi, \eta) z} \mathrm{~d} x \mathrm{~d} y \mathrm{~d} z}
$$

where $\mu$ and $k$ are the energy-dependent effective linear absorption coefficient and geometry factor, respectively. The geometrical weight function $w(x, y, z, \theta, \psi, \eta)$ describes the shape of the gauge volume (GV), which is that part of the sampling volume which lies inside the sample. The exponential term in equation (5) considers the attenuation of the x-ray beam by matter given by Beer's law.

For large-slit apertures in the primary and the diffracted beam, which are used in conventional XSA experiments, the shaping function $w(x, y, z, \theta, \psi, \eta)$ can be neglected. In this case the information depth only depends on the absorption factor and equation (5) takes the form $\langle z\rangle=\tau=1 / \mu \mathrm{k}$, which is called the $1 / e$ information depth. On the other hand, if narrow slits are used to define a very small sampling volume for high-resolution strain [23] or stress [24] scanning experiments in the real space, the absorption within the GV is usually neglected and the information depth is then given by the position of its geometrical centroid below the surface. The examples in this paper belong to the latter class of depth-profiling experiments.

\section{EXPERIMENT DETAILS}

The experiments presented in the following sections were carried out on the materials science beamline EDDI for ED diffraction, which the HelmholtzZentrum Berlin für Materialien und Energie has operated since 2005 at the synchrotron storage ring BESSY II. Technical details and performance data of the beamline have been reported in detail in reference [14]. The white synchrotron beam with usable photon energies between about $8 \mathrm{keV}$ and $100 \mathrm{keV}$ is provi-ded by a superconducting $7 \mathrm{~T}$ multipole wiggler. Therefore, in contrast to other materials science beamlines such as the ID15a at the ESRF in Grenoble or the new HEMS beamline at PETRA III in Hamburg, EDDI is a 'medium-energy' beamline with its main scientific focus on the characterization of structural gradients in the near surface region of materials and technical parts. Figure 2 shows the two-detector set-up which is now available at the EDDI beamline. It was realized by means of a $x-z$ translation stage at the back wall of the experimental hutch, which allows for a $z$-translation of the detector D0 within the vertical diffraction plane and for independent $x$ - and $z$-translations of the detector D1 in order to adjust diffraction conditions for simultaneous data acquisition out of the vertical diffraction plane.

In order to define a small sampling volume element, slit systems with small equatorial aperture are placed in front (S2') and behind (S3'/S3' ${ }^{\prime \prime}$ ) the sample, respectively as well as in front of both detectors (not labelled in Fig. 2). All slits are parallel to each other in order to ensure a small divergence, $\Delta 2 \theta$ (i.e. a high energy resolution in the diffraction patterns) and a high out-of-plane resolution for depth profiling. The sampling volume height can be adjusted between about $10 \mu \mathrm{m}$ and $50 \mu \mathrm{m}$ depending on the measuring problem $[\mathbf{1 6}, \mathbf{2 5}]$. The configuration with one entrance slit $\mathrm{S}^{\prime}$ ' for the diffracted beam(s) is applied to high-resolution depthprofiling experiments using two detectors, as will be demonstrated in section 4.1. In this case the equatorial slit S3' has to be placed as close as possible behind the sample to assure that both the beam diffracted into the detector D0 and that detected by detector D1 can pass this slit (case I in Fig. 2). Since that way both beams originate from the same GV, 


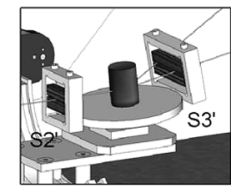

(I)

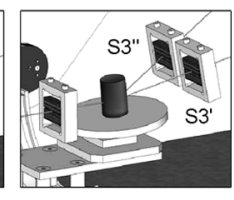

(II)

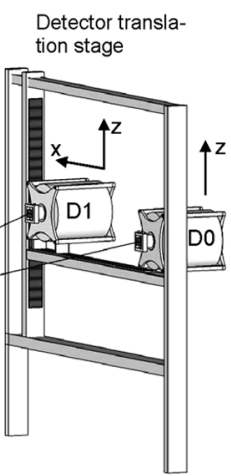

Fig. 2 Schematic view of the different detector set-ups available at the EDDI beamline for real space residual strain/stress and texture depth profiling and for in-situ thin-film stress analysis, respectively. Case (I): The diffracted beams analysed with the detectors D0 and D1 mounted in the $x-z$-translation stage $1.6 \mathrm{~m}$ behind the sample pass one and the same slit S3' (oneentrance-slit configuration). Case (II): The diffracted beams pass different slits S3' and S3' (two-entrance-slits configuration). For experiments employing the two-detector set-up, the $2 \theta$-detector arm of the basic diffractometer unit is in a parking position where it does not disturb the diffracted beams. See text for further details

this one-entrance-slit set-up can be used for simultaneous lattice strain analysis in different directions of the diffraction vector with respect to the gauge volume, that is, the sample reference system (see Fig. 1).

For in-situ ED diffraction experiments that require voluminous sample environments such as reaction chambers for thin-film processing, the distance between the two diffracted beams (recorded in D0 and D1) at the place where they pass the entrance slit S3' becomes too large for employing only one slit. This problem was solved by using a two-entrance-slit set-up (case II in Fig. 2) as demonstrated in section 4.2. However, this configuration is at the expense of (depth) resolution, because the positions of the GVs defined by slits S3' and S3' may diverge to a certain extent, since they have to be aligned separately.

Figure 3(a) displays the $\psi$-angle range which can be realized by means of a horizontal translation $x$ of the detector D1. Because a horizontal shift out of the vertical diffraction plane leads also to an increase of the diffraction angle $2 \theta_{1}$ for detector D1 (see Fig. 3(b)), careful calibration with stress-free
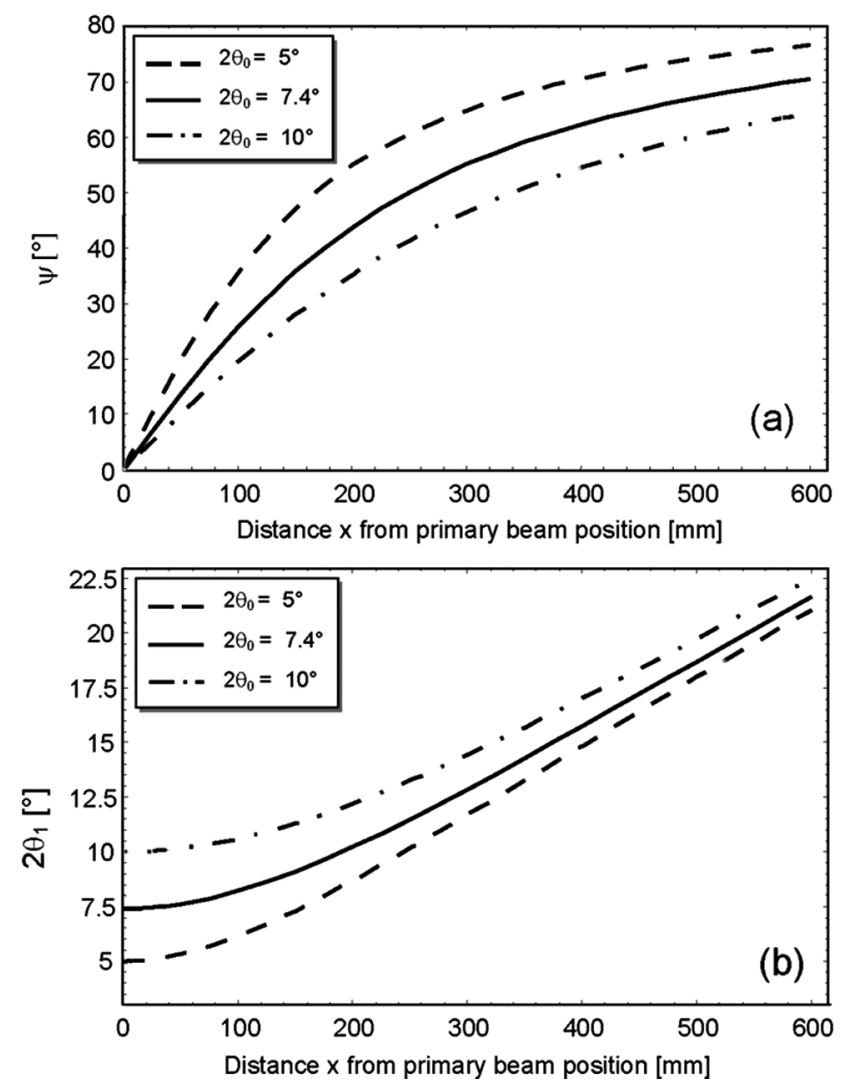

Fig. 3 Correlation between the $(x, z)$ position of detector D1 in the translation stage and the angle set $\left(2 \theta_{1}, \psi\right)$ which characterizes the diffraction geometry. The distance between the sample and the stage is $1600 \mathrm{~mm}$ and the point $(0,0)$ coincides with the position where the primary beam would hit the stage. The three diffraction angles $2 \theta_{0}$ of $5^{\circ}, 7.4^{\circ}$, and $10^{\circ}$ chosen for the calculations correspond to vertical distances $(0, z)$ from the primary beam of $z=140 \mathrm{~mm}, 208 \mathrm{~mm}$, and $282 \mathrm{~mm}$, respectively

standard powders is necessary to evaluate the exact $\left(2 \theta_{1}, \psi\right)$ angle set for each $(x, z)$ detector position.

\section{APPLICATION OF THE TWO-DETECTOR SET-UP TO ENERGY-DISPERSIVE STRESS AND TEXTURE ANALYSIS}

\subsection{Real-space depth profiling}

\subsubsection{Near-surface residual stress scanning in shot- peened steel}

The first example demonstrates the application of the one-entrance-slit set-up to high-resolution residual stress depth profiling in the $z$-space, which means that any information on lattice strains and residual stresses evaluated from the diffraction data 

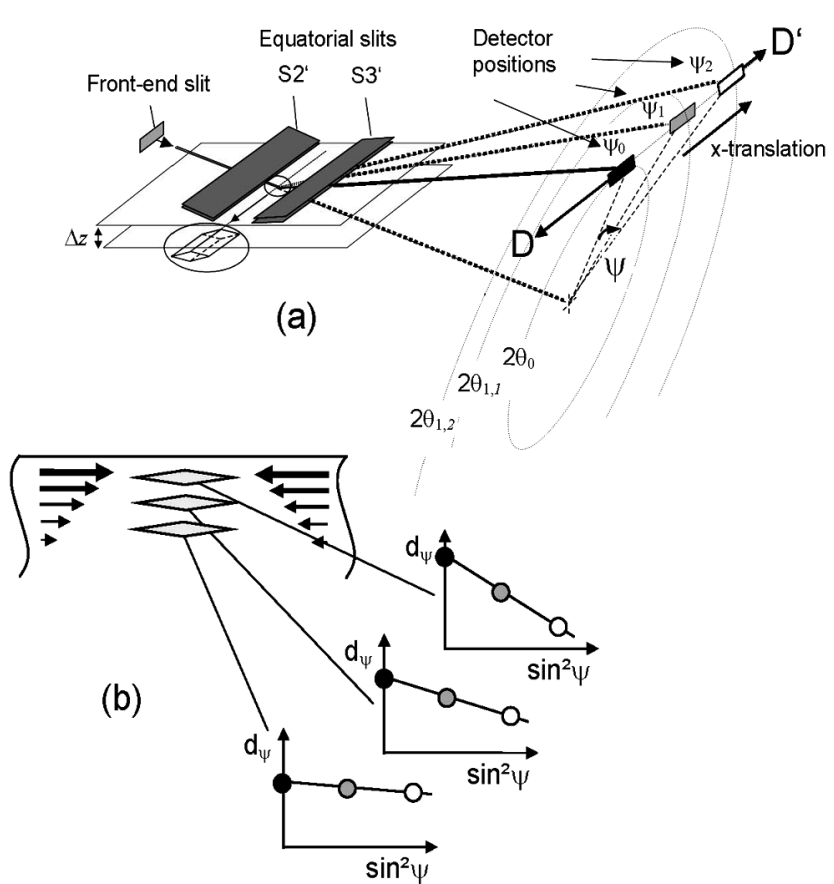

Fig. 4 Schematic view of the stress scanning method based on ED diffraction [24]: (a) geometrical set-up; and (b) principle of residual stress depth profiling in the $z$-space

is assigned to the centroid position $\langle\mathrm{z}\rangle$ of the gauge volume according to equation (5). The principle of this stress scanning method [24] may be understood by means of Fig. 4. From the sampling volume which is defined by the narrow slits, $\mathrm{S}^{\prime}$ and $\mathrm{S} 3^{\prime}$, diffracted intensity is scattered through the slit, S3' and intersects the detector translation stage in the horizontal line, $\mathrm{D}-\mathrm{D}^{\prime}$. According to the diagrams

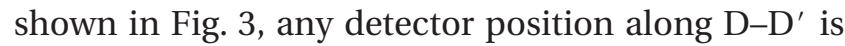
related to an angle set $\left(2 \theta_{1, i}, \psi_{i}\right)$. Starting from $\left(2 \theta_{0}\right.$, $\psi_{0}=0$ ), which corresponds to diffraction in the vertical plane, the measuring direction within the sampling volume can be varied by translating the detector along $\mathrm{D}-\mathrm{D}^{\prime}$ without changing the orientation of the gauge with respect to the sample system. Thus, strain depth scanning experiments can be performed without loss in spatial (i.e. depth) resolution for different $\psi$, which allows the use of the $\sin ^{2} \psi$ method to the $d_{\psi}^{h k l}$ data sets obtained in distinct depths $\langle z\rangle$ (see equation (5)) below the surface (see Fig. 4(b)).

Figure 5 shows the lattice strain and residual stress depth profiles obtained by applying the procedure outlined above to a roller bearing steel 100Cr6 in a hardened and tempered state, which was ground and subsequently shot-peened. The normalized strain depth profiles, $\varepsilon_{\psi}^{h k l}(z)$ in Fig. 5(a) were recorded for three horizontal detector positions and show a clear dependency on the respective inclination angle, $\psi$. In accordance with the residual stress gradient in Fig. 5(b), the effect of transverse contraction leads to positive (tensile) strains for $\psi=0^{\circ}$, whereas the negative (compressive) strains observed for $\psi=57^{\circ}$ evidence the direct impact of the compressive stress state. For $\psi=40^{\circ}$, which is close to the strain-free direction $\psi^{*}$ of the biaxial residual stress state given by $\sin ^{2} \psi^{*, h k l}=-2 S_{1}^{h k l} / \frac{1}{2} S_{2}^{h k l}$, the strain is about zero within the total investigated depth range, thus indicating the absence of pseudo-macro residual stress gradients in the normal direction, $\sigma_{33}$.

The residual stress depth profile in Fig. 5(b) was evaluated by applying the $\sin ^{2} \psi$ method to the individual strain data sets as shown in the inset panel. The results were verified by the layer removal method and show a distinct maximum of the compressive residual stresses about $30 \mu \mathrm{m}$ below the surface. At a depth of about $90 \mu \mathrm{m}$ the residual stresses change their sign into tensile residual stresses, which must balance the compressive residual stresses generated by the shot-peening treatment in the surface.

\subsubsection{Through thickness texture profiling in the Mg alloy AZ31}

The example introduced in this section is to illustrate how depth-resolved texture analysis can profit from the features of ED diffraction performed simultaneously with two detectors. For the investigations a wrought rolled AZ31 magnesium alloy was selected as a 'model' material because of the low absorption of Mg. The experiments reported here continue previous ED texture measurements carried out on the same sample using only the detector D0. The respective results showed that the ED method is well suited to detect the texture depth gradient over the sample cross-section which was introduced by bending the Mg alloy plate, for details see reference [16].

Since intensity depth profiling in reference [16] was performed without any sample tilt or rotation and only within the vertical diffraction plane, the accessible information on the crystallite orientation distribution was restricted to the normal direction of the sample. For pure fibre textures with rotational symmetry of the intensity distribution in any depth $z$, the analysis of complete diffraction patterns measured in the direction of the fibre axis would be sufficient to evaluate the texture with respect to both the fibre component(s) and its/their strength. In the present case, however, a more complex texture is to 

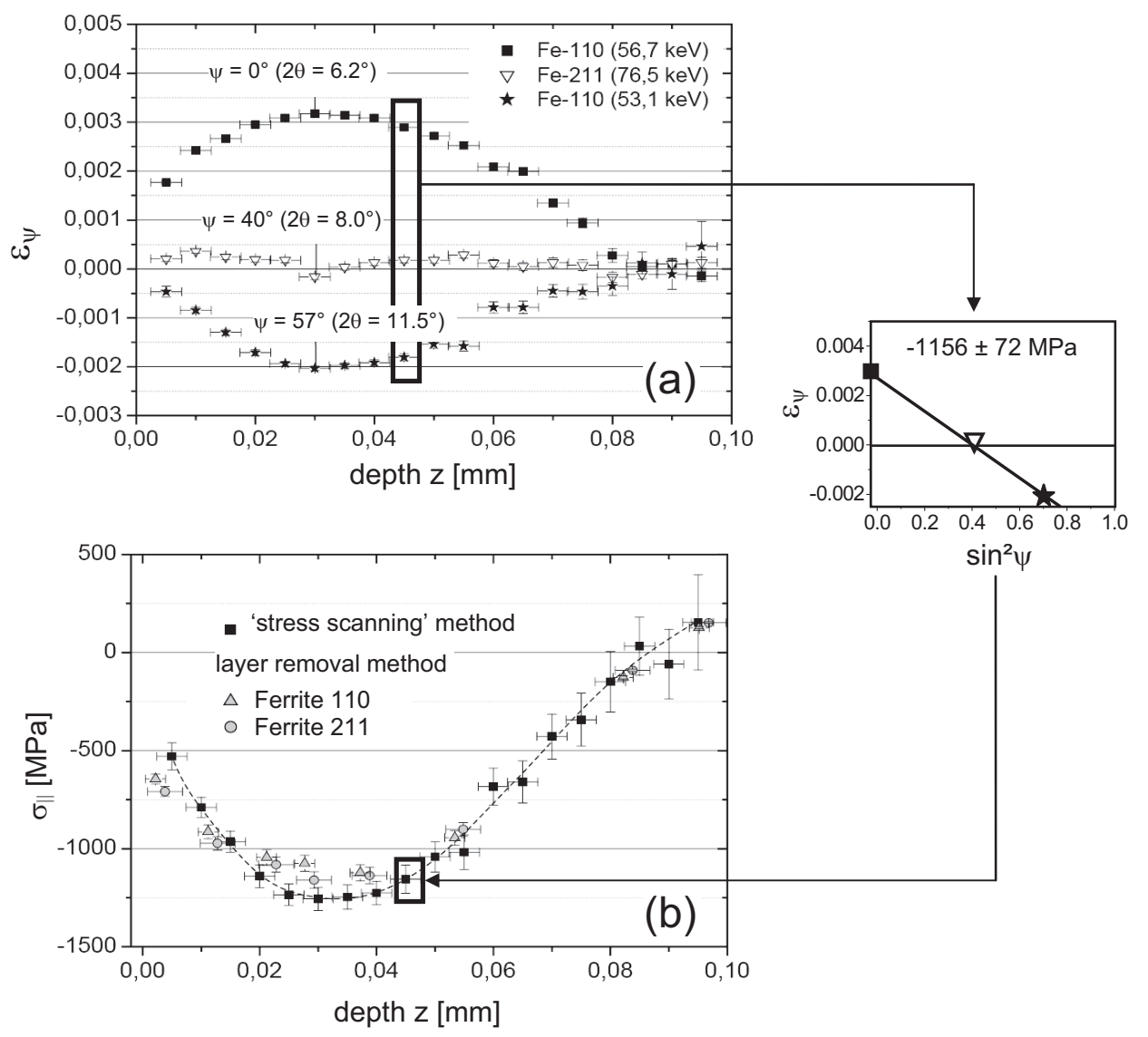

Fig. 5 ED residual stress analysis on a ground and subsequently shot-peened steel 100Cr6 employing the stress scanning method. The height of the sampling volume was $13 \mu \mathrm{m}$. (a) Normalized lattice strains $\varepsilon_{\psi}^{h k l}(z)$ obtained for three orientations $\psi$ which correspond to the $2 \theta$ angles in the brackets. Inset: example of the $\sin ^{2} \psi$ residual stress evaluation at fixed depth. (b) Real-space depth profile $\sigma(z)$ evaluated by means of the stress scanning method and the layer removal technique $[\mathbf{2 4}, \mathbf{2 6}]$

be expected which at least reflects the symmetry of the mechanical sample treatment in the form of uniaxial bending around an axis perpendicular to the normal plate. For this reason, in the second step of the investigation use was made of the two-detector set-up and the one-entrance-slit configuration in order to measure and analyse ED diffraction patterns in directions which are inclined by different angles, $\psi$ with respect to the normal sample surface. Figure 6 shows the sample geometry as well as an EBSD mapping performed on the cross-section, which clearly indicates the texture gradient generated by sample bending [16].

The measurements underlying the diagrams in Fig. 7 follow the same principle which already has been applied to the stress scanning method introduced in the previous section (see Fig. 4). The only difference is that in the present case the integrated intensities of the reflections $E^{h k l}$ are considered instead of the lattice strains. Therefore, the diagram in Fig. 7(b) represents to some extent the equivalent to the strain depth profiles shown in Fig. 5(a). From the intensity depth profiles in Fig. 7(b) it can be seen clearly that the increase in the integrated intensity of the $E^{0002}$ reflection from area I towards area II (see Fig. 6) becomes the stronger the smaller the inclination angle, $\psi$ between the normal sample and the measuring direction was chosen. This finding agrees well with the texture gradient analysed by means of the EBSD method (Fig. 6(b)), which

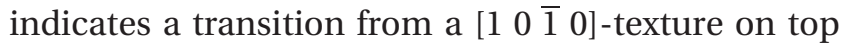

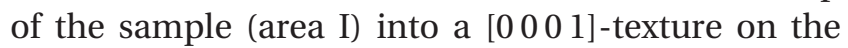
bottom (area II).

More detailed information on the orientation distribution, for example within the plane spanned by the sample normal $\left(\psi=0^{\circ}\right)$ and the rolling direction, $\operatorname{RD}\left(\varphi=90^{\circ}, \psi=90^{\circ}\right)$, can be obtained from a horizontal $x$-scan of the detector D1, which leads to a 


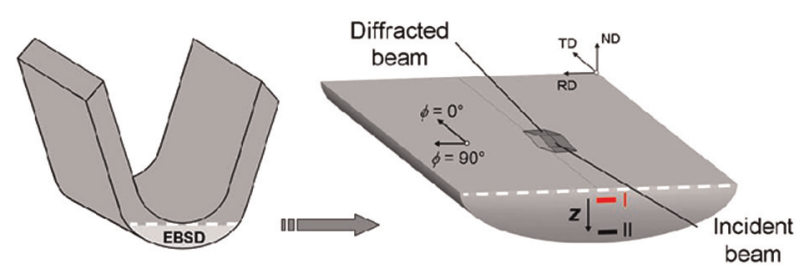

(a)

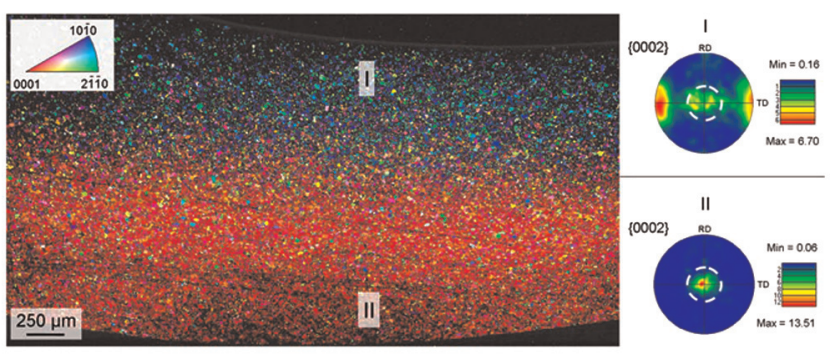

(b)

Fig. 6 (a) Schematic illustration of the bent AZ31 Mg alloy showing the region of interest for the ED texture studies. The two areas I and II mark the depths selected for the intensity diagrams shown in Fig. 7. (b) EBSD inverse pole figure map (left) and pole figures evaluated for the areas I and II (right). The dashed circles mark the inclination angle $\psi=30^{\circ}$ used for the $\varphi$-rotation scan in the depth areas I and II shown in Fig. 7(d). For more details on the texture distribution within the sample the reader is referred to reference [16]

variation of the inclination angle $\psi$ without changing the orientation between the sample and the gauge volume (see Figs 3 and 4). Figure 7(c) shows the respective results for the depth areas I and II. Although the covered $\psi$-range of about $20^{\circ}$ is rather small, it is to be seen that the considered $E^{0002}$ reflection is sensitive to the $\psi$-variation only in area II. Therefore, it reflects the $\left[\begin{array}{lll}0 & 0 & 1\end{array}\right]$ texture on the bottom of the sample. For area I, on the other hand, the intensity decrease is small compared to area II and has to be attributed mainly to geometrical effects and to the influence of the linear horizontal polarization of the synchrotron photons.

The $E^{0002}$-intensity distribution in Fig. $7(\mathrm{~d})$ was obtained from a $\varphi$-rotation scan performed at $\psi=30^{\circ}$. It displays the same twofold symmetry as to be seen in the pole figures in Fig. 6(b). However, the effect is significantly more pronounced for top area I which is characterized by the orientation relations $\left[\begin{array}{llll}1 & 0 & \overline{1} & 0\end{array}\right] \|$

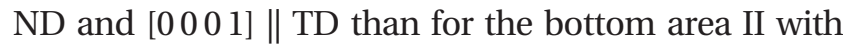
is closer to a real fibre texture $\left[\begin{array}{llll}0 & 0 & 0 & 1\end{array}\right] \| \mathrm{ND}$.

Finally, it has to be emphasized that intensity distributions as shown in Figs 7 (b) to (d) for the $E^{0002}$ reflection are available for all the reflections $E^{h k l}$ appearing in the diffraction patterns in Fig. 7(a).
Thus, an evaluation of complete ED diffraction patterns measured for various angle sets $(\varphi, \psi)$ using the method outlined above in different regions $z$ should allow in the future for an analysis of intensity as well as strain pole figures and, therefore, for combined depth-resolved texture and residual stress analysis.

\subsection{In-situ ED thin-film stress analysis using the two-detector set-up}

Another advantage of ED diffraction is considered in the following example. It consists in the possibility of doing diffraction experiments using a complex sample environment where it is difficult to 'thread' both the incoming and the diffracted beam(s) through the apparatus. Such experiments benefit from two features of high energy ED diffraction: (1) the measuring direction(s) can be chosen arbitrarily and therefore, in such a way that the beams can pass special entrance and exit windows of the devices; and (2) the high energy photons can penetrate even thick chamber walls and windows made, for example, of aluminium, which may simplify the device construction.

Figure 8 shows the experimental set-up used at the EDDI beamline for real-time studies on the rapid thermal sulphurization of $\mathrm{Cu}$-In thin film systems [27]. The ED-XSA investigations presented here were performed on a $500 \mathrm{~nm}$ thick Mo-film which serves as back contact for $\mathrm{Cu}(\mathrm{In}, \mathrm{Ga}) \mathrm{S}_{2}$ and $\mathrm{Cu}(\mathrm{In}, \mathrm{Ga}) \mathrm{Se}_{2}$ solar cells. The sample was produced by sputtering molybdenum on a glass substrate with a sputter pressure of $1 \mu$ bar. For in-situ stress analysis the sample was heated up in the vacuum chamber shown in Fig. 8 (b) to $500^{\circ} \mathrm{C}$ within $30 \mathrm{~min}$ and held in this condition for $5 \mathrm{~min}$. Subsequently, cooling down was done within $60 \mathrm{~min}$.

During the temperature cycle diffraction patterns were recorded with an integration time of $20 \mathrm{~s}$ employing the two-detector set-up and the 'twoentrance-slit' configuration shown in Fig. 8(a). From the diffraction patterns in Fig. 9(a) it can be seen that the larger diffraction angle used for data acquisition in detector D1 leads to a compression and shift of the diffraction lines towards smaller energies, whereas the position of the fluorescence lines remains unchanged. Stress evaluation on the basis of the $\sin ^{2} \psi$ method was performed for the Mo-110 reflection, which is the only one that appears with sufficient intensity under both diffraction conditions. Absolute calibration of the stress versus temperature curve was done by means of room temperature $\sin ^{2} \psi$ XSA measurements, which have been carried out before in the laboratory in the $\mathrm{AD}$ mode of diffraction. 

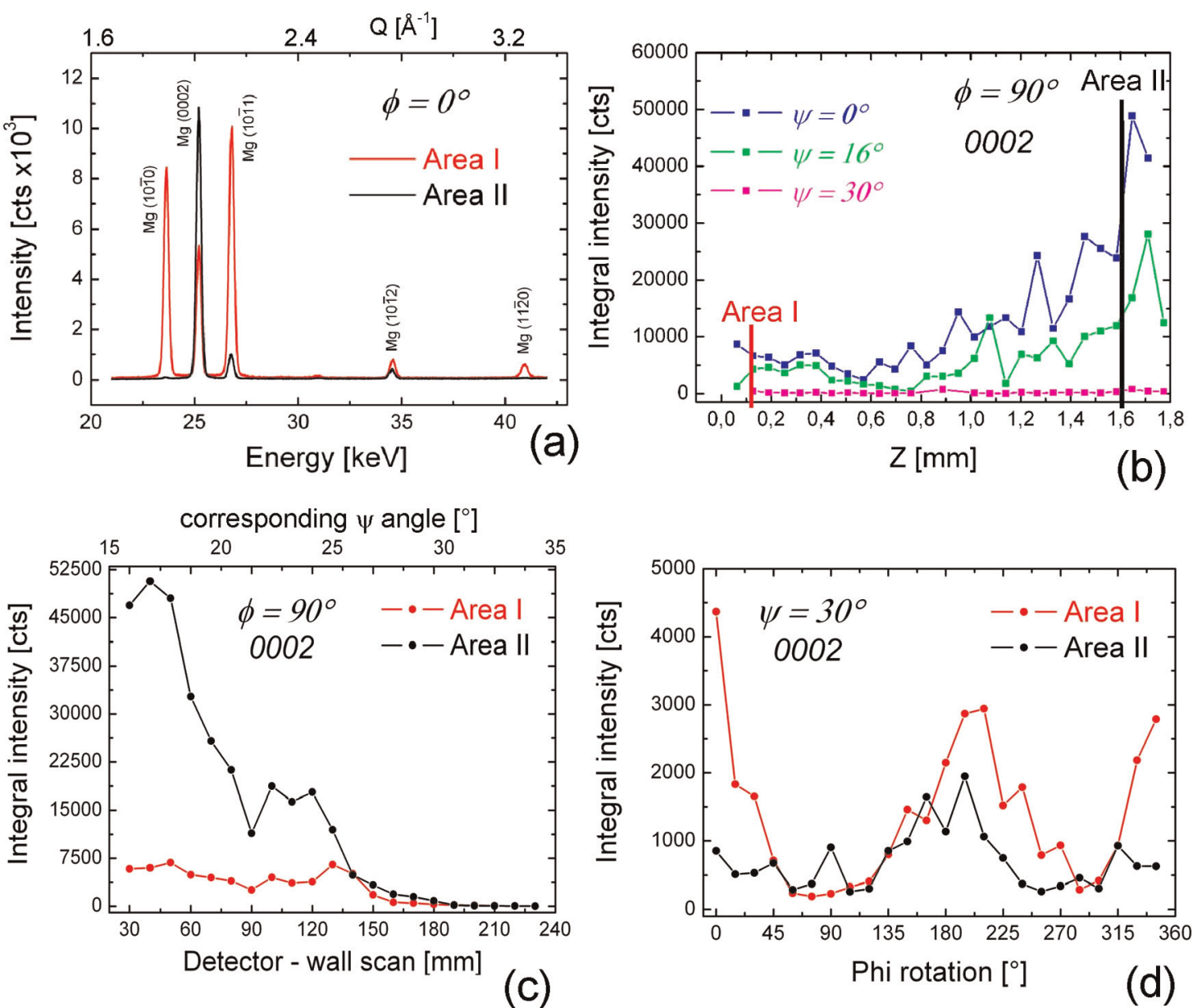

Fig. 7 ED texture investigations performed on the sample introduced in Fig. 6. (a) Diffraction patterns recorded under $2 \theta_{0}=9^{\circ}$ with detector D0 in the depth areas I and II marked in Fig. 6 . (b)-(d) Intensity distributions for the $E^{0002}$ reflection: (b) $z$-depth profiling with detector D0 $\left(2 \theta_{0}=9^{\circ}, \psi=0^{\circ}\right)$ and with detector D1 in two different horizontal positions which correspond to tilts of the diffraction vector towards RD of $\psi=16^{\circ}\left(2 \theta_{1}=9.24^{\circ}\right)$ and $\psi=30^{\circ}$ $\left(2 \theta_{1}=11.57^{\circ}\right)$, respectively; (c) horizontal $x$-scan with detector D1 in the depth areas I and II performed in the $\mathrm{RD}$ direction $\left(\varphi=90^{\circ}\right.$, see Fig. 6(a)); and (d) $\varphi$-rotation in the depth areas I and II recorded with detector D1 under $\psi=30^{\circ}$

The $\sigma_{\|}^{\mathrm{Mo}}(T)$ plot shown in Fig. 9(b) has to be discussed on the basis of the different thermal expansion coefficients of the Mo-film $\left(\alpha^{\mathrm{Mo}}=5 \cdot 10^{-6} \mathrm{~K}^{-1}\right)$ and the glass substrate $\left(\alpha^{\text {glass }}=9.5 \cdot 10^{-6} \mathrm{~K}^{-1}\right)$. Starting at room temperature from a high intrinsic compressive residual stress level of about $1400 \mathrm{MPa}$ which is due to the sputtering process, an onset of stress release is observed immediately after the beginning of the heating process. Then, the $\sigma_{\|}^{\mathrm{Mo}}(T)$ curve follows the temperature profile and reaches its maximum (i.e. the maximum value of stress release) at the temperature plateau of $500^{\circ} \mathrm{C}$, before it drops to about $-1150 \mathrm{MPa}$ at the final temperature in the process of $120^{\circ} \mathrm{C}$. Assuming a temperature dependency of $\Delta \alpha^{\text {Mo,glass }} \cdot E^{\mathrm{Mo}} \approx 1.5 \mathrm{MPa} \cdot \mathrm{K}^{-1}$ for the thermal film stress, extrapolation to room temperature would yield about $-1300 \mathrm{MPa}$, which is close to the result of $-1253 \pm 40 \mathrm{MPa}$ obtained afterwards by $\mathrm{AD}$-XSA performed in the laboratory. Furthermore, the maximum difference $\left|\sigma_{\|}^{\mathrm{Mo}}\left(T_{\max }\right)-\sigma_{\|}^{\mathrm{Mo}}(R T)\right|$ observed during the experiment, which is between about $700 \mathrm{MPa}$ and $900 \mathrm{MPa}$ (depending on whether the stresses after or before the process are considered, respectively), is in good agreement with the theoretical value of about $720 \mathrm{MPa}$ that is expected to arise for the temperature profile in the present heating cycle.

\section{CONCLUDING REMARKS AND OUTLOOK}

The ED diffraction method has a versatile field of applications, since it combines high flexibility regarding the multitude of possible experimental 

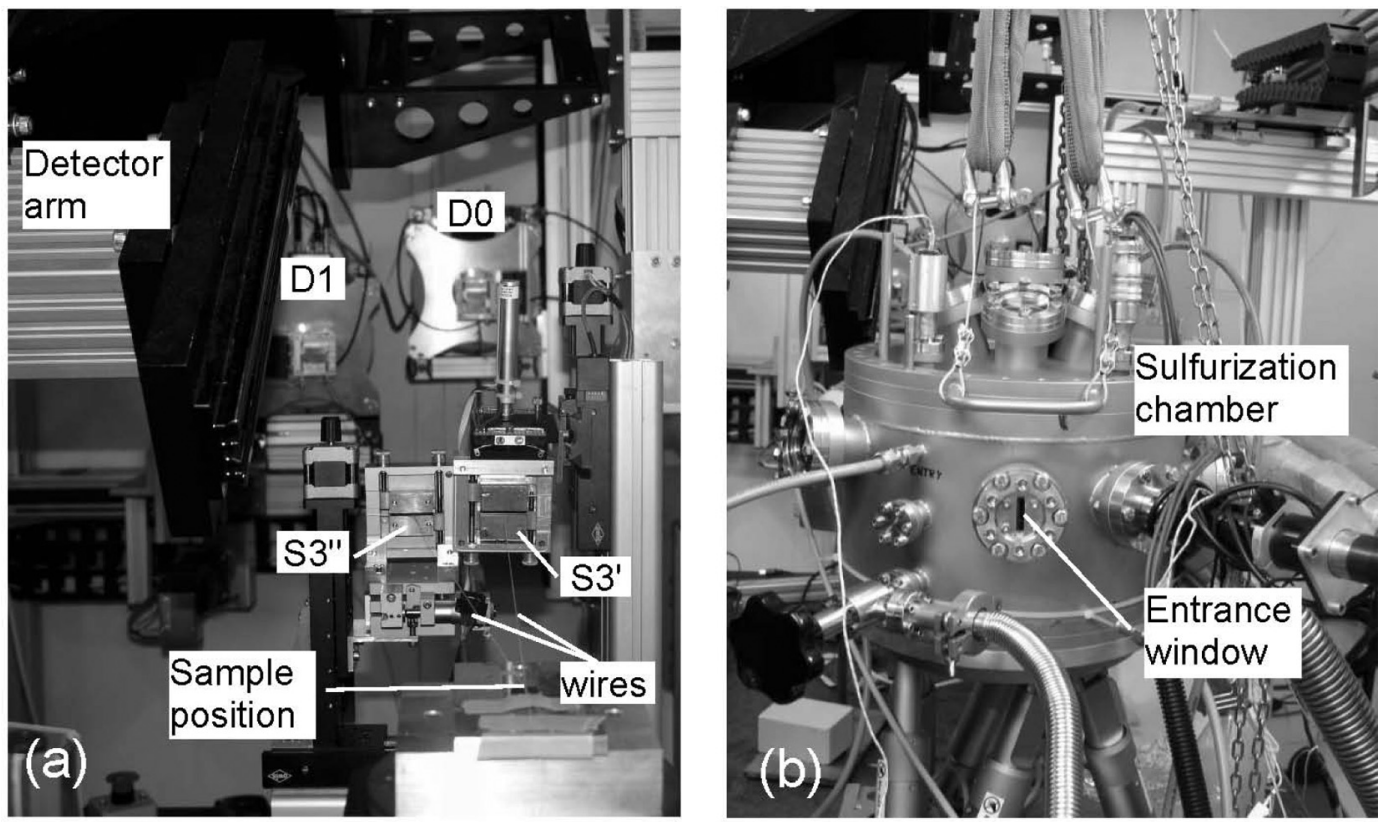

Fig. 8 (a) The two-entrance-slit-set-up at the EDDI beamline (see Fig. 2) for in-situ study of thinfilm reactions seen in propagation direction of the synchrotron beam. The two wires spanned between the sample and the detectors are used to align the slits S3' and S3' ${ }^{\prime \prime}$. (b) The reaction chamber for rapid thermal processing of thin films for solar cell fabrication is mounted on a hexapod stage in front of the secondary slit systems shown in (a)
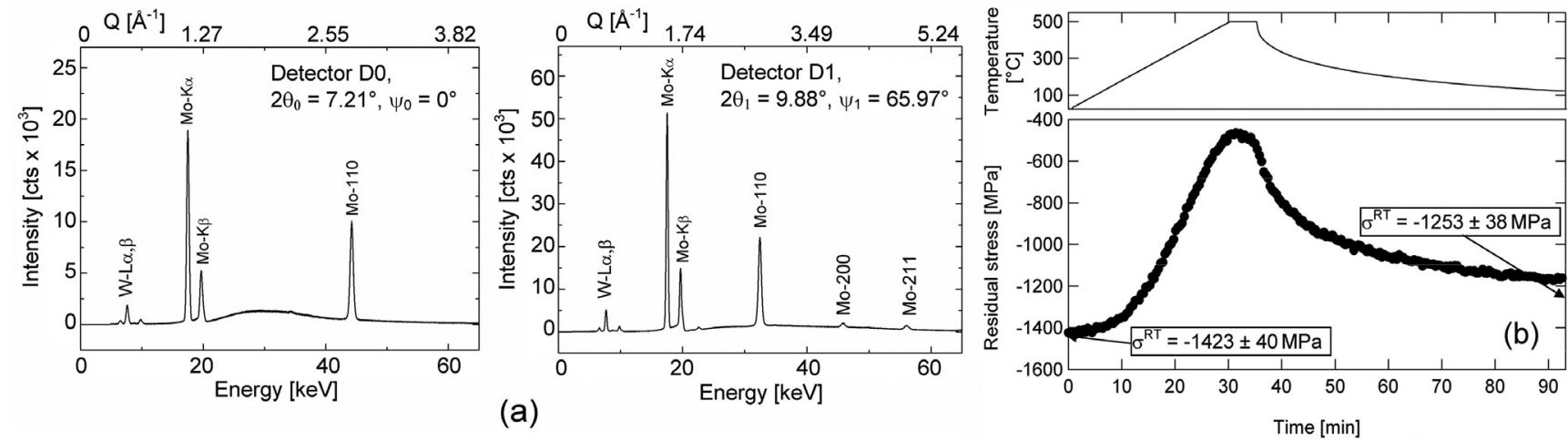

Fig. 9 (a) ED diffraction patterns recorded simultaneously with the detectors D0 and D1 for a 500 $\mathrm{nm}$ thick molybdenum film on a glass substrate. (b) $\sin ^{2} \psi$-based in-situ film stress analysis performed using the lattice spacings $d_{\psi=0^{\circ}}^{110}(T)$ and $d_{\psi=69.75^{\circ}}^{110}(T)$ which were evaluated from the diffraction patterns shown in (a). Evaluation of the exact angular positions $2 \theta_{0}$ (detector D0) and $\left(2 \theta_{1}, \psi_{1}\right)$ (detector D1) was done by means of stress-free $\mathrm{LaB}_{6}$ powder. $\sigma^{\mathrm{RT}}$ denotes the film residual stress measured at room temperature before and after the heating cycle in the $\mathrm{AD}$ diffraction mode in the laboratory

set-ups and configurations with a high information content contained in the diffraction patterns. In this paper a two-detector set-up which is now available at the EDDI beamline at BESSY II was introduced by means of examples from different fields of (residual) stress and texture analysis. It was demonstrated that the simultaneous acquisition of ED diffraction patterns with two detectors does not only mean a simple doubling of information but that it may result in a new quality of diffraction stress and texture analysis experiments.

Cases of residual stress and texture depth profiling in the real space were considered which are based on the same measuring principle. It consists in the use of two narrow slits in the primary and the diffracted beam path, respectively, in order to 
measure diffraction patterns with two detectors in different sample directions, which arise from the same small GV. Each ED pattern includes a multitude of diffraction as well as fluorescence lines containing full information on phase specific lattice strain, crystallographic texture, and the materials microstructure. Therefore, a future goal of ED diffraction analysis will be to merge high-resolution residual stress-, texture-, and microstructure depth profiling in one experimental and evaluation procedure. A significant challenge is to quantify exactly the orientation- and energy-dependent instrumental line broadening function, which is affected by both the diffraction geometry and the rather poor absolute intrinsic resolution of the ED solid state germanium detectors.

The second class of applications for the twodetector set-up are fast in-situ studies on thin-film growth processes. By the simple example of a single film on a glass substrate it was shown that it is possible to monitor in real time the stress evolution during a heat treatment. It is planned to extend these investigations to more complex multilayer systems and to take also into account texture and direction-dependent microstructure evolution within the individual sublayers of the coatings.

\section{FUNDING}

The authors are indebted to the German Research Foundation (DFG) for financial support of parts of the investigations, reference number GE 853/2-1, 2.

(c) Authors 2011

\section{REFERENCES}

1 Giessen, B. C. and Gordon, G. E. X-ray diffraction: new high-speed technique based on X-ray spectrography. Science, 1968, 159, 973-975.

2 Buras, B., Chwaszczewska, J., Szarras, S., and Szmid, Z. Fixed angle scattering (FAS) method for $x$-ray crystal structure determination, 1968, Report No. 894/II/PS (Institute of Nuclear Research, Warsaw).

3 Laine, E. and Lähteenmäki, I. The energy dispersive X-ray diffraction method: annotated bibliography 1968-1978. J. Mater. Sci., 1980, 15, 269-278.

4 Glazer, A. M., Hidaka, M., and Bordas, J. Energydispersive powder profile refinement using synchrotron radiation. J. Appl. Crystallogr., 1978, 11, 165-172.

5 Szpunar, J. and Gerward, L. Energy-dispersive xray diffraction studies of the texture in cold-rolled alpha-beta brass. J. Mater. Sci., 1980, 15, 469-476.
6 Gerward, L., Morup, S., and Topsoe, H. Particle size and strain broadening in energy-dispersive powder patterns. J. Appl. Phys., 1976, 47, 822-825.

7 Voskamp, A. P. High-speed retained austenite analysis with an energy dispersive $\mathrm{x}$-ray diffraction technique. Adv. X-ray Anal. 1974, 17, 124-138.

8 Buras, B., Olsen, J. S., Gerward, L., Will, G., and Hinze, E. X-ray energy-dispersive diffractometry using synchrotron radiation. J. Appl. Crystallogr., 1977, 10, 431-438.

9 Nagao, M. and Kusumoto, S. Poly-chromatic x-ray stress measurement. J. Soc. Mater. Sci. Jap., 1977, 26, 576-583 (in Japanese).

10 Ruppersberg, H. and Detemple, I. Evaluation of the complex stress field in a ground steel plate from energy dispersive x-ray diffraction experiments. Mater. Sci. Engng, 1993, A161, 41-44.

11 Reimers, W., Broda, M., Brusch, G., Dantz, D., Liss, K.-D., Pyzalla, A., Schmackers, T., and Tschentscher, T. Evaluation of residual stresses in the bulk of materials by high energy synchrotron diffraction. J. Nondestruct. Eval., 1998, 17, 129-140.

12 Steuwer, A., Santisteban, J. R., Turski, M., Withers, P. J., and Buslaps, T. High-resolution strain mapping in bulk samples using full-profile analysis of energy-dispersive synchrotron x-ray diffraction data. J. Appl. Crystallogr., 2004, 37, 883-889.

13 Genzel, Ch., Stock, C., and Reimers, W. Application of energy-dispersive diffraction to the analysis of multiaxial residual stress fields in the intermediate zone between surface and volume. Mater. Sci. Engng, 2004, A 372, 28-43.

14 Genzel, Ch., Denks, I. A., Gibmeier, J., Klaus, M., and Wagener, G. The materials science synchrotron beamline EDDI for energy-dispersive diffraction analysis. Nucl. Instrum. Methods Phys. Res., 2007, A578, 23-33.

15 Klaus, M., Reimers, W., and Genzel, Ch. Application of energy-dispersive diffraction to the analysis of highly inhomogeneous residual stress fields in thin film structures. Powder Diffr. Suppl., 2009, 24, S82-S86.

16 Coelho, R. S., Klaus, M., and Genzel, Ch. Throughthickness texture profiling by energy dispersive synchrotron diffraction. J. Appl. Crystallogr., 2010, 43, 1322-1328.

17 Brauser, S., Kromm, A., Kannengiesser, Th., and Rethmeier, M. In-situ synchrotron diffraction and digital image correlation technique for characterizations of retained austenite stability in lowalloyed transformation induced plasticity steel. Scr. Mater., 2010, 63, 1149-1152.

18 Huppmann, M. and Reimers, W. Development of twin fractions and micro stresses of the hot extruded magnesium alloy AZ31 under cyclic loading conditions. Mat. Sci. Forum, 2010, 638-642, 2411-2416.

19 Rodriguez-Alvarez, H., Mainz, R., Marsen, B., Abou-Ras, D., and Schock, H. W. Recrystallization of $\mathrm{Cu}-\mathrm{In}-\mathrm{S}$ thin films studied in situ by energy- 
dispersive x-ray diffraction. J. Appl. Crystallogr., 2010, 43, 1053-1061.

20 Thomas, D., Mainz, R., Rodriguez-Alvarez, H., Marsen, B., Abou-Ras, D., Klaus, M., Genzel, Ch., and Schock, H.-W. In-situ studies of the recrystallization process of $\mathrm{CuInS}_{2}$ thin films by energy dispersive x-ray diffraction. Thin Solid Films, 2010, accepted for publication.

21 Genzel, Ch. Evaluation of stress gradients $\sigma_{\mathrm{ij}}(z)$ from their discrete Laplace transforms $\sigma_{\mathrm{ij}}\left(\tau_{\mathrm{k}}\right)$ obtained by x-ray diffraction performed in the scattered vector mode. Phys. Stat. Sol. (a), 1996, 156, 353-363.

22 Xiong, Y.-S. and Withers, P. J. A deconvolution method for the reconstruction of underlying profiles measured using large sampling volumes. J. Appl. Crystal., 2006, 39, 410-424.

23 Withers, P. J. and Webster, P. J. Neutron and synchrotron x-ray strain scanning. Strain, 2001, 37, 19-33.
24 Denks, I. A. Entwicklung einer Methodik zur Erfassung randschichtnaher Eigenspannungsverteilungen $\sigma(z)$ in polykristallinen Werkstoffen mittels energiedispersiver Diffraktion. PhD Thesis, 2008, Universität Kassel, ISBN 978-3-89958-463-9.

25 Denks, I. A., Klaus, M., and Genzel, Ch. Determination of real space residual stress distributions $\sigma_{\mathrm{ij}}(z)$ of surface treated materials with diffraction methods. Part II: Energy dispersive approach. Mat. Sci. Forum, 2006, 524-525, 37-42.

26 Denks, I. A., Manns, T., Genzel, Ch., and Scholtes, B. An experimental approach to the problem of transforming stress distributions from the Laplace- into real space. Z. Kristallogr., 2009, Suppl. 30, 69-74.

27 Rodriguez-Alvarez, H., Kötschau, I. M., and Schock, H.-W. Pressure-dependent real-time investigations on the rapid thermal sulfurization of $\mathrm{Cu}-\mathrm{In}$ thin films. J. Crystal Growth, 2008, 310, 3638-3644. 